332 Kolbe: Rücksichtslosigkeit chemischer Autoren

hältnissen mit schwefliger Säure tief rothe Flüssigkeiten geben, die auf Zusatz von Salzen aller Art unlösliches Selen abscheiden. Es scheint demnach auch das Selen fähig zu sein, in colloiden Modificationen zu existiren.

Freiberg i. S., Laboratorium der Bergakademie.

\title{
Rücksichtslosigkeit chemischer Autoren gegen Verleger und das kaufende Publikum.
}

Wer einmal Wislicenus' Lehrbuch der organischen Chemie in die Hand bekommt, den befremdet beim ersten Blick die Raumvergeudung, welche der Autor durch den Missbrauch mit seinen chemischen Formeln und Gleichungen sich hat zu Schulden kommen lassen. Hier ein paar Beispiele als Belege:

Auf Seite 304 des Lehrbuchs nimmt die Formel des Triäthylbiurets, welche hier in natürlicher (oder vielmehr unnatürlicher) Ausdehnung wiedergegeben ist,

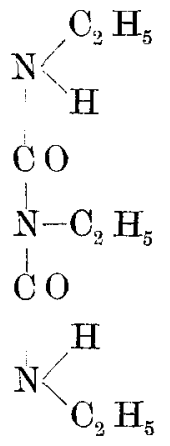

den Raum von 14 Zeilen ein, consumirt also, da die Seite 42 Zeilen hat, genan ein Drittheil der ganzen Columne.

Solcher Raum verschwendender Formelbilder finden sich Hunderte in den 73 Druckbogen. - Seite 75 und 77 führen nur je drei Zeilen, S. 74 und 81 nur je zwei Zeilen Text, 
gegen Verleger und das kaufende Publikum. 333 der übrige Raum ist mit unnöthig breiten Formelbildern gefüllt. Auch auf S. 79 sieht man nichts als Formeln.

Welcher Missbrauch mit den übermässig lang gedehnten Formel-Bindestrichen getrieben wird, lehrt u. A. folgendes auf S. 136 stehende, noch dazu sinnlose Formelbild für das Mellon:

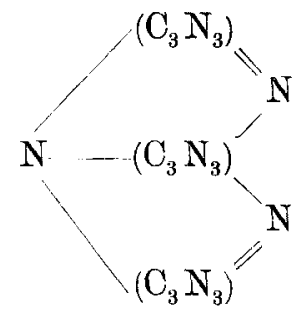

Man geht gewiss nicht fehl in der Behauptung, dass in jedem Exemplar dieses dickleibigen Buches mehr als fünf Druckbogen an Löschpapier hätten gespart werden können, was sicher im Interesse des Verlegers und des kaufenden Publikums gelegen haben würde.

Im grossartigen Maassstabe betreibt unerhörte, sinnlose Raumvergeudung ein anderer Chemiker, Hr. Michaelis, in seiner Bearbeitung des Graham-Otto'schen Lehrbuches der anorganischen Chemie. Derselbe erreicht das auf zweierlei Weise, einmal dadurch, dass er, wie die grossen, so auch die kleinsten, unwichtigsten Capitel welche oft nicht mehr als drei Zeilen einnehmen, mit mächtigen, aus grösster und fettester Schrift gewählten Ueberschriften versieht (vergl. Bd. II, Abth. II, S. 1037), sodann dadurch, dass er die Zwischenräume, vom Schlusse des einen Capitels bis zum Anfang des folgenden, in ungebührlicher Weise ausdehnt (vgl. S. 670, wo vom Schluss des Capitels "Bor und Schwefel", welches nicht ganz eine Druckseite füllt, bis zum Anfange des nächsten Capitels „Bor und Stickstoff" mehr als eine Druckseite leer gelassen ist).

Michaelis, welcher auf diese und in noch viel schlimmerer W eise die Raumverschwendung in's Grosse treibt, kann gegenüber den damit nicht zu vergleichenden, geringeren 


\section{Kolbe: Räcksichtslosigkeit chemischer Autoren}

Vergehungen von Wislicenus, mit Franz Mohr sagen: „Ich habe mich nie mit Kleinigkeiten abgegeben."

Die folgenden Blätter legen dar, dass der eben angegebene Fall nicht vereinzelt dasteht.

Man schlage Bd. II, Abth. II auf, wo Seite 1246 bis 1248 Niobverbindungen behandelt sind. Das erste dieser Blätter bringt zunächst sieben Zeilen Text als Schluss des auf der voraufgegangenen Seite begonnenen Capitels Nioboxychlorid; dann folgt ein neuer, mit der Ueberschrift "Nioboxybromid" versehener vierzeiliger Abschnitt, hierauf ein neunzeiliger Abschnitt mit der Ueberschrift „Nioboxyfluorid."

Damit, nämlich mit-20 Zeilen Text und zwei Ueberschriften, wird die eigentlich fünfzigzeilige Columne (1246) als gefüllt erachtet. Der Rest ist unbedruckt gelassen. Die zwei folgenden Seiten 1247 und 1248 sind jede weitaus nicht zur Hälfte mit Druckerschwärze versehen. Die erste derselben hat nur 15 Zeilen und zwei Ueberschriften, deren eine, „Niob und Schwefel", mit grösster fetter Schrift gesetzt ist. - Die zweite Seite hat 19 Zeilen Text und eine mächtige Ueberschrift: „Niob und Stickstoff".

Zur Aufnahme von zusammen 53 Zeilen Text auf den drei Seiten 1246-1248, und von fünf Ueberschriften, wofür der Raum von $1 \frac{1}{2}$ Columnen ( 75 Zeilen) genügt hätte, beansprucht Hr. Michaelis den doppelten Raum, drei Columnen.

Man ist im Zweifel, was man mehr bewundern soll, das Geschick, womit Michaelis das zu Wege bringt, oder die Unverfrorenheit, womit er dem chemischen Publikum und dem Verleger solches bietet.

Auf S. 1239 ist das Capitel "Tantal und Stickstoff" behandelt. Dasselbe besteht aus dieser, mit fettester Schrift gesetzten Ueberschrift und nur 18 Zeilen Text; dazu ist aber die ganze Columne in Anspruch genommen. Ueber die Hälfte derselben steht leer.

Seite 1121 enthält nichts als die zwei mächtigen Ueberschriften „Molybdän und Jod" und „Molybdän und 
gegen Verleger und das kaufende Publikum. 335

Fluor", erstere mit fünf, letztere mit acht Zeilen Text, also zwei Ueberschriften mit zusammen 13 Zeilen Text. Auf der nächsten Seite folgt die Rubrik "Molybdän und Sauerstoff". Bis zu dieser Ueberschrift ist abermals der Raum von zehn Druckzeilen leer gelassen.

Auf S. 1134 und 1135 vermittelt den Uebergang vom Ende des Capitels „Molybdänsäure und Molybdändioxy d" zum nächsten Capitel „Molybdün und Schwefel" nahezu eine ganze leere Columne.

Auf Bogen 16, S. 256 schliesst das Capitel „Untersalpetrige Säure“ mit neun Zeilen Text; der Platz für die übrigen 41 Zeilen ist nicht bedruckt.

Auf Bogen 42, S. 670 stehen gar nur acht Zeilen Text des Capitels: "Bor und Schwefel". Um für den Uebergang zum nächsten Capitel: "Bor und Stickstoff" Athem zu schöpfen, lässt $\mathrm{Hr}$. Michaelis auf S. 670 nicht weniger als 42 Zeilen und auf S. 671 noch 10 Zeilen unbedruckt, zusammen mehr als eine Columne.

Auf Bogen 75 sind Seite 1196-1199 vier Capitel mit den Ueberschriften: „Uranoxysulfid", „Uran und Stickstoff", "Zur Bestimmung und Scheidung des Urans" und "Nebengruppe des Stickstoffs" behandelt. Der von diesen vier Ueberschriften dominirte Text besteht in Summa aus 80 Zeilen. Rechnen wir auf jede der vier Ueberschriften den Raum von fünf Zeilen Text, also zusammen 20. Zeilen, so ergiebt sich, dass zur Aufnahme der ganzen vier Capitel der Raum von 100 Zeilen, d. i. zwei Druckseiten ausgereicht haben würde.

Hr. Michaelis hat es durch unsinnige Platzvergeudung fertig gebracht, dafür den doppelten Raum, vier Columnen, $\mathrm{zu}$ verwenden.

Auf S. 1165, welche nur 16 Zeilen Text führt, und auf S. 1166 mit der grossen Ueberschrift: „Wolfram und Phosphor"s und mit 19 Zeilen Text, also im Ganzen mit 40 Zeilen, füllt Hr. Michaelis zwei Columnen, vergeudet also wieder eine Columne.

Auf S. 1006 ist, ganz unmotivirt, der Raum von 27 Zeilen, auf S. 1009 der von 28 Zeilen, auf S. 1017 der von 32 Zeilen, 
336 Kolbe: Rücksichtslosigkeit chem. Autoren etc.

auf S. 994 der von 33 Zeilen, und auf S. 1027 gar der von 38 Zeilen leer geblieben; was auf den beiden Seiten 1026 und 1027 steht, hätte auf einer Columne überreichlich Platz gehabt. Hier fällt die grandiose Raumvergeudung besonders deutlich in's Auge.

Am tollsten hat Michaelis mit dem zu bedruckenden Raume der Seiten 1037-1040 gewirthschaftet. - Auf S. 1037 sind nicht weniger als drei Capitel erschöpfend behandelt, nämlich: „Thorium und Schwefel" mit 12 Zeilen Text, sodann "Thorium und Stickstoff" mit fünf, und "Thorium und Phosphor" mit gar nur drei Zeilen Text. Der gesammte Text auf S. 1037 füllt also 20 Zeilen. Der Raum für die übrigen 30 Zeilen wird von jenen drei, mit grösster, fetter Schrift gesetzten Ueberschriften consumirt.

Seite 1038 steht leer.

Seite 1039 enthält nichts, als die fünf Worte:

Die Nebengruppen der Nichtmetalle.

Seite 1040 ist .... leer.

Die sieben Seiten: 1037-1043 haben weder Seitenzahlen, noch Columnentitel!

Jedem wird sich wohl die Frage aufdrängen: Was veranlasst Herrn Michaelis zu solch' colossaler, den Verleger, wie das Bücher kaufende Publikum schädigender Raumvergeudung in seiner Bearbeitung des Graham-Otto'schen Lehrbuches?
Leipzig, im April 1883.
H. Kolbe. 